\title{
Philosophiques
}

\section{Introduction : Cosmopolitisme et particularisme}

\section{Jocelyne Couture et Kai Nielsen}

Volume 34, numéro 1, printemps 2007

Cosmopolitisme et particularisme

URI : https://id.erudit.org/iderudit/015860ar

DOI : https://doi.org/10.7202/015860ar

Aller au sommaire du numéro

Éditeur(s)

Société de philosophie du Québec

ISSN

0316-2923 (imprimé)

1492-1391 (numérique)

Découvrir la revue

Citer ce document

Couture, J. \& Nielsen, K. (2007). Introduction : Cosmopolitisme et particularisme. Philosophiques, 34(1), 3-15. https://doi.org/10.7202/015860ar d'utilisation que vous pouvez consulter en ligne.

https://apropos.erudit.org/fr/usagers/politique-dutilisation/ 


\title{
Introduction : Cosmopolitisme et particularisme
}

\author{
JOCELYNE COUTURE \\ KAI NIELSEN
}

En proposant un volume sur le cosmopolitisme et le particularisme, nous avons voulu mettre en évidence une opposition qui, à notre avis, a joué et continue de jouer un rôle structurant dans les débats qui animent la philosophie politique contemporaine. Dans ce qui suit, nous voulons montrer que cette opposition, d'abord conçue comme une dichotomique tranchée, a forcément évolué, à la faveur des débats, vers des positions plus nuancées de part et d'autre jusqu'à ce que ses multiples déclinaisons en vienne à toucher, en plus des problèmes soulevés par la présente conjoncture mondiale, une grande partie des questions traditionnelles de la philosophie politique. Les contributions réunies dans le présent volume en donnent un exemple frappant. Nous ne les discuterons pas en détail ; elles sont suffisamment claires et brèves pour n'avoir besoin ni d'explication ni de résumé. Nous pourrons nous y référer au passage, mais notre objectif dans cette introduction est plutôt d'expliciter le contexte philosophique dans lequel elles prennent place.

I.

Le cosmopolitisme est une ancienne doctrine dont on attribue la paternité aux stoïciens, surtout romains. Elle repose à son origine sur l'idée d'un ordre moral universel, accessible à tous par la raison et composé des principes, des devoirs et des obligations que chaque être humain doit mettre en pratique à l'endroit de chacun de ses semblables. Sénèque et Marc-Aurèle défendent l'idée que, même si chacun est membre d'une communauté locale, c'est la communauté mondiale des êtres humains qui est la source de nos obligations et de nos valeurs les plus importantes. Nous sommes, disent-ils, «citoyens du monde ». Les philosophes des Lumières ont repris à leur compte cette doctrine, et Kant l'a formalisée dans un idéal de justice cosmopolitique qui devrait s'incarner, selon lui, dans une fédération mondiale de républiques, garante d'une paix perpétuelle et respectueuse de la dignité de chacun.

Bien que la doctrine cosmopolitique ait été reprise sous des formes diverses, c'est en tant que doctrine morale qu'elle intéresse surtout les philosophes ${ }^{1}$. Dans ses versions contemporaines, elle reprend essentiellement les idées anciennes, mettant de l'avant le point de vue voulant que l'allégeance morale première d'une personne aille à la communauté mondiale des êtres humains et affirmant que nous avons les mêmes devoirs et les mêmes

1. Les philosophes s'intéressent surtout à la théorie idéale du cosmopolitisme et accordent très peu d'attention à ce que certains anthropologues appellent «le cosmopolitisme réel » qu'ils observent dans le monde actuel. Selon ceux-ci, il y aurait trois figures du cosmopolitisme : 1) Le polyglotte instruit, qui voyage beaucoup, qui se sent chez lui partout, à l'aise dans toutes

PHILOSOPHIQUES 34/1 — Printemps 2007, p. 3-16 
obligations à l'endroit de chacun, quels que soient son origine, sa nationalité ou l'endroit du monde où il vit. D'où l'opposition des défenseurs du cosmopolitisme à toute forme de particularisme, à commencer par le nationalisme et le communautarisme. Nous ne devrions pas, selon eux, accorder de primauté morale aux membres d'une communauté locale telle que la nation, ou à nos coreligionnaires, ou à une famille politique. Au cœur de cette exigence, on trouve ainsi une croyance purement morale voulant que tous les humains aient une valeur égale, que la vie de tous compte et compte également, et que chaque être humain, du moins initialement, mérite un égal respect.

Le cosmopolitisme implique minimalement une certaine posture morale en matière de justice. L'idée d'une charte des droits universels est d'inspiration cosmopolitique, de même que celle d'une justice distributive à l'échelle mondiale. Les défenseurs du cosmopolitisme se font aussi très souvent les avocats d'interventions interétatiques en cas de violations des droits de la personne. S'ils n'ont pas le monopole des conceptions de la justice globale, ils en sont des partisans convaincus, se désintéressant d'une façon caractéristique des conceptions plus étroites qui se limiteraient, par exemple, à définir les arrangements institutionnels souhaitables au sein de certains types d'organisation étatique. Pour les défenseurs du cosmopolitisme, ce qui est moralement souhaitable pour les uns doit également l'être pour tous. Et les bénéficiaires premiers des mesures de justice doivent inconditionnellement demeurer les individus et non les groupes, les peuples et encore moins les États. Parmi les philosophes contemporains qui ont défendu cette position, Martha Nussbaum est sans doute la plus représentative ${ }^{2}$.

Mais d'autres avocats du cosmopolitisme ont soutenu que la consistance de cette doctrine appelait impérativement, davantage qu'une simple posture morale, la création d'un cadre institutionnel spécifique à l'échelle internationale. Pour eux, David Held en particulier ${ }^{3}$, le système étatique actuel, fondé sur la souveraineté des peuples, divise les « citoyens du monde ", crée des inégalités entre eux et il est un frein intolérable à l'épanouissement d'une mentalité, d'une justice et d'une démocratie authentiquement cosmopolitiques. Il propose donc une dispersion de la souveraineté vers des instances supranationales, régionales ou locales reconfigurées, favorisant selon lui une meilleure participation des individus aux décisions qui les touchent et mieux en mesure de contribuer à

les cultures et qui n'a d'allégeance ni de racines nulle part. Cette figure est incarnée par certains artistes et par les grands aventuriers. 2) Les cadres des sociétés multinationales détenteurs de MBA des grandes universités et leurs assistants haut gradés voyageant d'une métropole à l'autre, connaisseurs des gastronomies nationales et menant, de façon générale, un train de vie flamboyant. 3) La grande majorité des travailleurs migrants, œuvrant et vivant dans des endroits très différents de leur lieu d'origine et souvent avec d'autres ouvriers venant aussi de lieux très différents. (1) et (2) illustrent des formes élitistes du « cosmopolitisme réel » et une indifférence bienveillante à l'endroit de l'humanité qu'on a parfois reproché aux philosophes d'idéaliser dans leur description des "citoyens du monde».

2. Voir par exemple Nussbaum, 2002.

3. Held, 1995. 
l'égalité et à la liberté de chacun quels que soient son origine, son rang social et sa nationalité. Si les États ne disparaissent pas entièrement de ce modèle institutionnel, ils en sont néanmoins réduits à jouer un rôle surtout administratif dans une structure de gouvernance partagée. Le modèle proposé par Held soulève plusieurs problèmes dont le moindre n'est pas d'évoquer ce que Kant craignait, à savoir un gouvernement mondial ${ }^{4}$.

Pour une autre figure influente du cosmopolitisme, Charles Beitz, cette doctrine n'a aucune implication politique ou institutionnelle. Beitz écrit que «le cosmopolitisme ne recèle aucun présupposé concernant la meilleure structure politique pour les affaires internationales; qu'il doive y avoir une organisation politique globale et, si oui, la façon dont l'autorité doit être répartie entre l'organisation globale et ses éléments subordonnés constitue réellement un problème pour la science politique normative et non pas pour la philosophie ellemême ${ }^{5}$.» David Miller, tout en pensant que Beitz a tort, le voit comme un digne « représentant de la façon dont on comprend le cosmopolitisme en philosophie contemporaine $»^{6}$. Bien qu'il s'agisse probablement là d'une exagération, il faut néanmoins reconnaître que des philosophes comme Martha Nussbaum, Kwame Anthony Appiah et Samuel Scheffler affirment que ce qui est vraiment en jeu dans le cosmopolitisme est une posture morale, et ils en discutent en des termes purement moraux ${ }^{7}$. Ce n'est pas le cas des philosophes qui ont contribué au présent volume et qui se dissocient également, d'une façon générale, du cosmopolitisme institutionnel de David Held. Certains d'entre eux, cependant, voudront préserver le caractère individualiste, propre au cosmopolitisme, des normes de la justice globale, reprenant ainsi à leur compte l'idée que les nations, contrairement aux individus, n'ont aucune pertinence morale (voir, par exemple, Mollendorf dans le présent ouvrage).

Miller souligne un problème pour les défenseurs du cosmopolitisme, et ce problème en est aussi un pour certains de nos auteurs. Miller pense que les théoriciens du cosmopolitisme se rendent coupables d'un non sequitur « lorsqu'ils disent que chaque humain a une valeur morale égale et affirment que, par conséquent, nous devons traiter tous les humains également au sens où nous aurions les mêmes devoirs envers tous ${ }^{8}$. Un parent, par exemple, doit aider son enfant dans ses travaux scolaires, mais il serait bien implausible de prétendre qu'il a le devoir d'aider aussi tous les enfants du monde. Une personne a des obligations à l'endroit d'un ami qui sont différentes de celles qu'elle a à l'endroit d'un parfait étranger. Nous avons des obligations

4. Pour une critique du modèle institutionnel de D. Held, voir Couture, 1999, et Habermas, 2001.

5. Beitz, 1998, 83.

6. Miller, 2002, 81.

7. Nussbaum, 2002. Samuel Scheffler a montré comment et pourquoi il en est ainsi, Appiah, 2005, et Scheffler, 2001.

8. Miller, 2002, 82. Thomas Pogge a répondu à la critique que fait Miller du cosmopolitisme; voir Pogge, 2002b. 
envers nos collègues que nous n'avons pas à l'endroit de ceux qui ne le sont pas. Cela fait tout simplement partie de la pratique qui consiste à avoir des enfants, des amis, des collègues, pratiques qu'une société a toutes les raisons de valoriser ${ }^{9}$. Les cosmopolites qui, avec raison, considèrent que tous les êtres humains ont une égale valeur ne devraient pas pour autant conclure que nous avons les mêmes devoirs à l'endroit de chacun. Rejeter cette forme de cosmopolitisme ne signifie pas que nous rejetons l'égale valeur morale de tous, mais cela signifie que nos devoirs doivent être différenciés; nous devons plus à certains qu'à d'autres.

Cela conduit Miller (et aussi Scheffler) à distinguer entre un cosmopolitisme faible et un cosmopolitisme fort. Le cosmopolitisme faible pose que tous les êtres humains ont une égale valeur morale. C'est la conception que Miller et bien d'autres considèrent comme étant vraie ou appropriée (selon leur méta-éthique), mais plusieurs la considèrent aussi comme un truisme. Nul, du moins depuis la chute du fascisme et de l'apartheid, ne l'a ouvertement contestée. Le cosmopolitisme, dans sa version forte, est la doctrine voulant que chaque humain doit traiter tous les autres de la même manière et que les devoirs qu'il a envers les uns il les a envers tous. Selon Miller, cette doctrine est platement fausse. Ainsi, le cosmopolitisme est souvent présenté comme étant ou bien un lieu commun ou bien une fausseté.

Les auteurs qui, dans le présent volume, défendent le cosmopolitisme ont-ils trouvé une façon de parer à cette conclusion ou de rejeter à la fois ce que Miller appelle le cosmopolitisme faible et le cosmopolitisme fort? Sans nous faire juge de cela, nous voulons pourtant souligner qu'il y a là un problème auquel tous les théoriciens du cosmopolitisme doivent faire face.

II.

Dans ses derniers travaux, John Rawls propose une conception de la justice globale ${ }^{10}$ que plusieurs s'entendent à qualifier d'anti-cosmopolitique. Ce verdict s'appuie sur le fait que cette théorie focalise sur les peuples ${ }^{11}$, contredisant ainsi le dictum cosmopolitique voulant que les individus, en tant qu'êtres humains, doivent être l'objet premier de nos préoccupations morales. Ce déplacement a pour effet que Rawls refuse d'étendre à la justice globale plusieurs des principes qu'il défendait à l'échelle de la justice domestique, comme le principe de différence, le principe d'égalité des chances et le principe de la valeur équitable des libertés politiques. À la place de ces principes qui garantissaient des droits et des libertés individuels, on retrouve, dans la théorie de la justice globale rawlsienne, huit principes régissant les relations

9. Samuel Scheffler a exploré ces raisons dans Scheffler, 2001.

10. Rawls, 1999.

11. S'il est vrai que la théorie de Rawls focalise sur les peuples, il faut néanmoins souligner le fait que, pour lui, ce sont les frontières entre les États qui déterminent ce qui compte pour un peuple. C'est la raison pour laquelle certains commentateurs (par exemple, Wenar dans le présent volume) ont pu parler de l'étatisme de Rawls. 
internationales et les traités. Font partie de la Société des peuples et sont, par conséquent, régies par ces principes, les nations libérales et démocratiques, mais aussi ce que Rawls appelle les sociétés décentes, c'est-à-dire des hiérarchies consultatives de type patriarcal qui ne sont ni agressives ni expansionnistes. Toutes ces nations ont un devoir d'assistance à l'endroit des sociétés « éprouvées ", c'est-à-dire des sociétés qui, en raison de circonstances historiques, sociales et économiques, peinent à réaliser leur autonomie politique ${ }^{12}$. Le devoir d'assistance se limite strictement à l'obligation d'aider les sociétés éprouvées à se donner et à maintenir des institutions justes ou décentes. Pour Rawls, il n'y a aucun principe de justice distributive exigeant de combler l'écart entre les sociétés riches et les sociétés pauvres au delà de ce que demande le principe d'assistance.

Peu ont applaudi à la parution de The Law of Peoples où Rawls présente cette conception de la justice globale. Comme le dit Leif Wenar dans le présent volume: «La Société des peuples, telle que la conçoit Rawls, exige d'une part que les peuples soient justes selon les paramètres de leurs propres conceptions de la justice, et ce, dans les limites de la légitimité politique et, d'autre part, qu'ils se comportent entre eux comme de bons voisins. » Mais en dépit de ceux qui ont pensé reconnaître dans cet ouvrage un virage conservateur ou une parodie de la justice globale ${ }^{13}$, il importe de comprendre, comme Wenar nous y invite ici, les raisons pour lesquelles Rawls se montre apparemment si peu exigeant à l'endroit des nations qu'il inclut dans la Société des peuples et pourquoi, en particulier, il se démarque des exigences du cosmopolitisme.

Nous identifions pour notre part deux raisons, qui convergent vers ce qui semble être un impératif de la justice globale rawlsienne, à savoir l'absence de domination entre les peuples. La première tient à la légitimité des principes qui régissent les rapports de justice entre les nations. Dans tout l'œuvre de Rawls, la question de la légitimité apparaît comme une préoccupation centrale. Des principes de justice institutionnalisés, que ce soit à l'échelle globale ou dans le cadre d'un État, ont force de loi et supposent par conséquent l'exercice d'un pouvoir coercitif. Or, pour Rawls et pour toute la tradition du libéralisme politique, le pourvoir coercitif n'est légitime que si les principes qui gouvernent les institutions sont jugés acceptables par ceux qui le subissent. Il semble assez clair ici que des principes de justice et des institutions " cosmopolitiques » conçues pour régir les relations entre les personnes à l'échelle de la planète contreviendraient de façon flagrante à ce qu'il pourrait sembler raisonnable d'accepter. Et cela, non seulement parce qu'ils iraient à l'encontre du statu quo en matière de système étatique et de relations internationales, comme certains l'ont suggéré, mais aussi pour des raisons purement organisationnelles: les conventions légales ou semi-légales qui régissent la vie publique dans chaque pays, qu'il s'agisse de la famille, de la santé ou de la sécurité, ne peuvent tout simplement

12. Rawls, 1999, 117.

13. Voir par exemple Pogge, 1994, 2002a, et Nussbaum, 2005. 
pas s'effacer devant des règles régissant les relations d'individu à individu. Or ces conventions soulèvent des questions de justice sociale qui doivent être réglées à l'échelle d'une société de la façon qui semble acceptable aux membres de cette société.

En limitant les exigences imposées aux peuples par la justice globale, Rawls se soucie de la légitimité du pouvoir coercitif exercé sur les peuples libéraux et décents dans leurs relations entre eux. Les institutions mondiales de la justice ne peuvent pas légitimement exiger de ces peuples qu'ils signent tous les traités, abolissent leurs frontières, congédient leurs armées et partagent entre eux leur richesse tant qu'il ne leur apparaît pas raisonnable de le faire. Et Rawls se soucie également de la légitimité du pouvoir coercitif qui s'exerce au sein des États libéraux et décents lorsqu'il s'agit de se donner et de faire respecter des principes de justice sociale. Les institutions mondiales de la justice ne peuvent pas légitimement forcer un gouvernement à se donner des politiques d'égalité des chances, à remodeler ses institutions ou à adopter certains modes de développement, car ces décisions, chez les peuples libéraux et décents, doivent s'appuyer sur le consentement du peuple et dépendent de l'acquiescement de celui-ci aux valeurs qui sous-tendent ces pratiques.

Cela nous amène à la seconde raison qui a pu conduire Rawls à limiter les exigences de la justice globale. Peu de temps après la parution de Théorie de la justice, Rawls a commencé à émettre des réticences quant au projet de définir des principes de justice à valeur universelle. Dans Libéralisme politique, déjà, il souligne la difficulté de créer un consensus sur le deuxième principe de justice dans une société libérale. Selon lui, le pluralisme qui règne dans ces sociétés de même que la profonde divergence des intérêts qu'on y rencontre pourraient faire obstacle à son acceptation générale. Rawls insiste aussi sur les difficultés épistémiques (les difficultés de la raison) qui coupent court aux prétentions à la vérité en matière de justice. Ces préoccupations ne sont bien sûr pas très éloignées de celles qui touchent à la légitimité. Mais elles approfondissent cette question en mettant l'accent sur les conditions du consentement. En particulier, elles attirent l'attention sur le fait que de multiples facteurs peuvent influencer les points de vue sur la justice et que ces facteurs ne sont pas tous moraux. Nous pensons que, s'agissant de la justice globale, Rawls a pu vouloir inclure parmi ces facteurs la culture, les traditions et les coutumes des peuples libéraux et décents. Tout comme le pluralisme au sein d'une société libérale, le pluralisme à l'échelle internationale nous oblige à réduire la portée des principes de justice.

L'inclusion, dans la Société des peuples, de sociétés qui ne sont ni démocratiques (ou si peu), ni égalitaristes, ni libérales mais seulement "décentes » a soulevé l'indignation de plusieurs commentateurs qui ont vu dans la mince justice globale de Rawls une concession faite à ces peuples. Ils ont peut-être raison. Il est assez clair qu'entre le danger de trop concéder et celui d'imposer à la planète des valeurs, des modes de vie marqués au coin par l'Occident, c'est le second que Rawls craignait le plus. Mais cette crainte, à notre avis, ne se 
fonde pas uniquement sur une exigence de légitimité; elle prend aussi sa source dans la reconnaissance du fait qu'il y a plusieurs façons pour un peuple de concevoir ses institutions, les rapport entre ses membres, son évolution, ses modes de vie et son développement, bref de se concevoir lui-même, et que certaines de ces façons, même si elles diffèrent profondément des nôtres, peuvent néanmoins être "décentes ». Pour le dire autrement, l'imposition à tous des mêmes normes de justice pourrait elle-même constituer une injustice. Et reconnaître cela c'est aussi reconnaître que les peuples libéraux et décents ont la capacité de décider par et pour eux-mêmes ${ }^{14}$.

\section{III}

Ces deux raisons de limiter les exigences de la justice globale — un souci pour la légitimité et pour l'autodétermination des peuples — devraient intéresser les défenseurs du cosmopolitisme. Ce sont elles, en effet, qui alimentent l'une des critiques les plus tenaces adressée au cosmopolitisme sous toutes ses formes, des plus anciennes aux plus récentes, à savoir la ressemblance de famille qu'il entretient avec l'impérialisme et le colonialisme. Le colonialisme s'est toujours présenté sous les couleurs d'une mission civilisatrice. Prétextant de l'égalité de tous les êtres humains, et par conséquent de l'admissibilité de tous à recevoir la bonne nouvelle, le colonisateur s'est aussitôt empressé de reléguer le colonisé dans un rôle d'apprenti devant son maître. L'idée que chaque personne a des devoirs moraux à l'endroit de l'humanité tout entière est peutêtre une bonne nouvelle pour certains, mais qui définira le contenu de ces devoirs et les droits qui y correspondent ? Qui, au nom de l'égalité de tous, s'arrogera la tâche d'enseigner au reste de l'humanité ce qui est bon pour elle? Qui, sans être à la fois juge et partie, aura le mandat de soumettre le genre humain à la norme ? Par delà ce qui ressemble bien à une aporie de l'égalité cosmopolitique, on retrouve ici deux problèmes proprement moraux que Rawls, croyons-nous, avait bien aperçus : celui de la légitimité des normes et celui de l'autodétermination des personnes et des peuples. Mais ces problèmes ne sont-ils pas, finalement, inhérents à toute tentative d'asseoir les fondements d'une justice globale substantielle?

Dans ce volume, Maria Herrera s'attaque à cette question et elle y apporte une réponse qui pourra sembler être un compromis inacceptable aux yeux de certains théoriciens du cosmopolitisme, à savoir, le contextualisme. C'est, selon elle, en se plaçant du point de vue des cultures particulières et de leurs rapports historiques avec les autres nations que l'on peut identifier les

14. Nous ne voudrions pas occulter ici les difficultés pratiques de la position de Rawls. Comme l'ont fait remarquer plusieurs commentateurs, c'est souvent au nom du peuple et de son bien que la tyrannie et l'oppression finissent par s'imposer. Mais il faut aussi se rappeler que le respect des peuples prôné par Rawls vaut uniquement pour les peuples libéraux et décents qui font partie de la Société des peuples et non pas pour les régimes expansionnistes et ceux qui violent les droits de la personne. Le pari que fait Rawls est qu'en les incluant dans la Société des peuples, les peuples décents se libéraliseront. 
raisons de chacun, non seulement de souscrire à certaines valeurs mais aussi d'acquiescer à l'établissement d'un ordre mondial juste et contraignant. On peut penser, en effet, que ce n'est que lorsqu'un consensus se dégage au terme d'un tel processus que l'on peut vraiment parler de valeurs universelles. Mais cette position tranche avec l'hypothèse, chère aux défenseurs du cosmopolitisme mais aussi à un grand nombre de philosophes depuis Kant, voulant que la source de telles valeurs soit une rationalité "désengagée » et universellement partagée.

Herrera n'est pas la seule ni la première à soutenir que le cosmopolotisme requiert une forme de particularisme. Il y a dix ans déjà, Mitchell Cohen parlait de la nécessité, pour la compréhension des idéaux et le développement d'une sensibilité cosmopolitiques, d'un "ancrage » dans l'expérience d'une communauté humaine particulière ${ }^{15}$. Nous avons séparément soutenu qu'un nationalisme "réitératif » était compatible avec le cosmopolitisme ${ }^{16}$. Dans le présent volume, Kok-Chor Tan soutient que le nationalisme implique un égalitarisme universaliste. Comment les défenseurs de ce néocosmopolitisme composent-ils avec les vices qu'attribuaient leurs prédécesseurs à toute forme de particularisme, à l'étatisme, à la priorité accordée aux compatriotes, aux identités locales?

D'abord, en souscrivant à un nationalisme libéral et en se dissociant fermement de toute forme de nationalisme ethnique, exclusif, fondé sur la filiation ancestrale ou la supériorité de la race, que les théoriciens du cosmopolitisme ont avec raison dénoncé. L'inconvénient est qu'en condamnant sans phrases le nationalisme, ils n'avaient souvent en tête que cette forme dévoyée du nationalisme. Une exception à cela, Brian Barry, qui, prenant acte du concept, soutient que le nationalisme libéral "has zero examplification». Pourtant le nationalisme libéral est celui qu'on retrouve, entre autres, en Écosse, en Catalogne et au Québec. Il épouse, comme son nom l'indique, les valeurs du libéralisme politique, à savoir l'égalité, la tolérance, les libertés fondamentales, le respect des minorités nationales et la démocratie. Et surtout, il est itératif au sens où, si le principe de nationalité ${ }^{17}$ vaut pour une nation libérale, alors il vaut pour toutes les nations libérales. Ce qui requiert des nationalistes, non seulement qu'ils respectent les autres nations libérales et leurs aspirations mais aussi qu'ils les soutiennent dans la poursuite de leurs projets.

Un défenseur du cosmopolitisme pourrait toujours objecter que le nationalisme, même s'il est libéral et itératif, constitue une enfreinte à l'égale considération due à tous les êtres humains puisqu'il commande forcément d'accorder

15. Cohen, 1995.

16. Couture, 2002, Nielsen, 1999.

17. Il existe plusieurs caractérisations du principe de souveraineté. La plus répandue est celle voulant que chaque nation, ou bien possède son propre État, ou bien fasse partie d'une authentique fédération de nations (État multinational). Dans ce dernier cas, le principe exige qu'une nation possède ses propres institutions et qu'elle contrôle elle-même son propre développement et l'évolution de sa culture, bref qu'elle soit en mesure de s'autodéterminer. 
la priorité aux devoirs que nous avons envers nos compatriotes. Nous avons parlé plus haut des devoirs cosmopolitiques et nous avons conclu, avec Miller, que l'idée que nous ayons les mêmes devoirs envers tous les habitants du globe manquait de plausibilité. Mais une variante plus plausible de ce problème ressurgit lorsqu'on aborde la question de l'immigration et de l'ouverture des frontières ${ }^{18}$. Si l'on songe qu'une grande part de l'immigration dans les pays riches provient des pays économiquement défavorisés, il semble évident que la justice et l'égalité des personnes commandent une ouverture des frontières plus grande que celle qu'on observe présentement. Une ouverture totale des frontières, que certains théoriciens du cosmopolitisme pourraient vouloir favoriser, n'est pas possible en pratique pour des raisons qui tiennent à la capacité des pays, même riches, de faire face à une augmentation massive et accélérée de leur population. Un nationaliste libéral accepterait que ses frontières soient plus ouvertes qu'elles ne le sont dans la plupart des pays; mais il voudra néanmoins éviter que le flux de l'immigration ne vienne submerger la culture et la langue nationales. Mais n'est-ce pas là un exemple flagrant d'une injustice endossée par les nationalistes libéraux au nom de la priorité qu'ils accordent à leurs compatriotes? N'est-ce pas là la preuve, a contrario, que les défenseurs du cosmopolitisme ont raison de s'opposer aux particularismes?

Dans sa contribution au présent volume, Tan soutient que l'efficacité des pratiques d'édification de la nation auxquelles se livrent tous les États libéraux en vue de maintenir et d'alimenter une culture et une identité nationales suppose un certain contrôle de l'immigration. Mais il argue aussi qu'un nationalisme libéral et itératif appelle nécessairement des mesures de justice distributive égalitariste à l'échelle mondiale puisqu'il implique l'obligation de créer, pour les autres nations libérales, les conditions matérielles de leur autonomie politique.

Tan a montré, dans un autre ouvrage ${ }^{19}$, comment les pratiques dans lesquelles une nation s'engage pour protéger sa culture et sa langue peuvent être justifiées d'un point de vue moral. Ses arguments s'inspirent en bonne partie de celui de Will Kymlicka voulant que l'appartenance culturelle constitue un bien premier essentiel au développement des capacités morales d'une personne et, en premier lieu, de son identitée ${ }^{20}$. Si l'appartenance culturelle est un bien premier pour les personnes, il s'ensuit que le nationalisme itératif est un principe cosmopolitique.

L'argument de Kymlicka ne le conduit pas à cette conclusion mais plutôt à défendre une politique de multiculturalisme visant la reconnaissance, au sein des États libéraux, des groupes culturels et des minorités nationales et la provision, pour ces groupes, de droits différenciés. Selon certains défenseurs du cosmopolitisme, si la position de Kymlicka sur ce point illustre la dérive des identités locales coupées du reste de l'humanité, pour

18. Nous avons traité de cette question dans Couture et Nielsen, 2005.

19. Tan, 2000.

20. Kymlicka, 1989. 
d'autres elle tendrait plutôt vers une essentialisation des cultures et des identités nationales, finalement préjudiciable aux membres des groupes ciblés. Dans le présent volume, Jocelyn Maclure dénonce cette dernière tendance (bien qu'il en exonère Kymlicka) et soutient que la reconnaissance des groupes n'a pas à faire appel à l'essentialisme identitaire ou culturel que réprouvent les défenseurs du cosmopolitisme. Comme pour étayer encore cette position, Pablo Gilabert soutient ici que c'est le point de vue de la justice globale, et non pas celui des identités ou des cultures, qui doit éclairer les questions relatives à la citoyenneté et au multiculturalisme et, en particulier, la question de la reconnaissance des groupes minoritaires à l'intérieur des États. Tan et Gilabert, montrent donc chacun à leur manière que l'universalisme moral est non seulement compatible avec la reconnaissance des devoirs différenciés à l'endroit de certains groupes particuliers mais qu'il est requis pour une conceptualisation et une identification adéquates de ces devoirs.

\section{Conclusion}

Le débat entre les avocats du cosmopolitisme et ceux du particularisme mobilise, comme nous venons de le voir, plusieurs enjeux de la philosophie politique. S'il est une question, cependant, qui a cessé de figurer au programme, c'est bien celle de savoir quelle partie l'emporte sur l'autre. En effet, et comme le montre bien l'ensemble des contributions réunies dans cet ouvrage, le débat se cristallise de plus en plus sur la façon d'intégrer des éléments de la doctrine cosmopolitique aux points de vue particularistes et vice versa. Concrètement, ces tentatives s'inscrivent dans le projet de concilier les exigences de la justice domestique et celles de la justice globale. Si la perspective cosmopolitique force naturellement l'attention sur des questions de justice globale, il est de plus en plus clair que celle-ci ne peut pas s'articuler indépendamment de la justice sociale ni en nier la pertinence. La principale raison à cela est que la justice requiert, sur les deux plans, des institutions qui devront s'arrimer entre elles pour jouer efficacement leur rôle. De plus, comme Rawls l'a amplement souligné, la forme des institutions régissant les relations entre les peuples de même que la participation de ceux-ci aux institutions de la justice globale dépend dans une large mesure des formes d'organisation sociale qu'ils se sont données.

Il est tout aussi difficile d'imaginer que la justice globale pourrait consister en une simple extension, à l'échelle mondiale, des théories de la justice domestique, comme le croient certains rawlsiens convertis aux vertus du cosmopolitisme. Rawls, encore une fois, mais aussi Kant, en ont fait valoir les raisons morales. Cette conception du cosmopolitisme confond l'idéal d'égalité avec l'uniformisation de tous dans une structure mondiale unifiée. De plus, il nous paraît utopique et sans doute conceptuellement erroné de penser la Société des nations sur le modèle d'une société étatique. Prise littéralement, l'expression "citoyen du monde" n'a aucune réalité et ne devrait avoir aucun avenir. 
S'écartant de leurs formes les plus radicales et les plus idéalisées, les valeurs cosmopolitiques tendent, pour l'heure, à se traduire dans des doctrines diverses qui vont de l'humanisme à l'internationalisme en passant pas l'égalitarisme et l'universalisme moral. Ces doctrines ne se réduisent pas les unes aux autres, mais elles n'ont généralement pas les implications résolument antiparticularistes et parfois anti-étatiques du cosmopolitisme initial.

De l'autre côté, si les avocats du particularisme tirent souvent argument de la valeur morale des cultures, des solidarités locales et des communautés humaines, ils ont montré de façon convaincante que ces valeurs sont instrumentales et que l'objet premier de leurs conceptions morales demeurait la personne. Cette position les rapproche grandement de l'individualisme cosmopolitique. Par ailleurs, la conjoncture mondiale actuelle ne peut que leur donner raison de porter leur attention sur des questions de justice globale qui touchent directement ou indirectement leurs positions. Les effets de la mondialisation de l'économie, tels l'apparition d'agences de gouvernance globale non démocratiques, l'uniformisation des cultures, l'affaiblissement de la souveraineté des peuples, soulèvent autant de problèmes de justice globale qui commandent l'adoption d'une perspective cosmopolitique.

Tout comme pour le cosmopolitisme, les idéaux du particularisme tendent maintenant à s'exprimer dans des doctrines diverses. Celles-ci, comme le libéralisme compréhensif, le contextualisme ou le nationalisme itératif ne se réduisent pas les unes aux autres, mais elle n'ont pas les implications antiégalitaristes, et parfois exclusivistes, d'un particularisme strict.

Est-ce à dire que le débat est clos et qu'aucune divergence substantielle ne subsiste entre une perspective particulariste et une perspective plus cosmopolitique? Nous ne le croyons pas. L'une des difficultés que présente la doctrine cosmopolitique, comme le montre bien l'article de Ryoa Chung dans le présent volume, est de comprendre ce qu'elle requiert exactement en matière de droits, d'obligations, de principes de justice et d'institutions. Les défenseurs du cosmopolitisme ne s'entendent d'ailleurs pas à ce sujet. On a reproché à certains d'entre eux de ne défendre qu'une posture morale somme toute sans conséquence pour la réalisation des idéaux cosmopolitiques. Mais d'autres, comme nous l'avons souligné, se sont attaqués au problème en esquissant le modèle institutionnel mondial requis, selon eux, par la réalisation de ces idéaux. S'ils ont raison sur ce point, alors on doit s'attendre à des discussions vigoureuses entre les défenseurs des doctrines particularistes et ceux qui défendent ce modèle, et peut-être même entre ces derniers et ceux qui défendent une autre forme de cosmopolitisme institutionnel.

Ce n'est pas ici le lieu d'entreprendre ce débat, mais nous pouvons néanmoins en circonscrire les termes. Les défenseurs du cosmopolitisme institutionnel se répartissent en deux catégories. Premièrement, il y a ceux, dont nous avons déjà parlé, qui pensent que la réalisation des idéaux du cosmopolitisme requiert le remplacement, à l'échelle internationale, des structures d'organisation sociale et politique telles que nous les connaissons maintenant par des 
institutions plus souples, décentralisées, au sein desquelles les "citoyens du monde " pourront former, selon leurs intérêts, des associations. Ce modèle implique donc la disparition — ou la dissolution jusqu'à l'insignifiance - du système étatique, des frontières, de la souveraineté nationale et des institutions traditionnelles de la démocratie. Deuxièmement, il y a ceux qui pensent que les idéaux cosmopolitiques peuvent être réalisés en conservant le système étatique que nous connaissons, mais requièrent la création d'institutions internationales et supra-nationales capables de faire appliquer certains principes de distribution et de veiller au respect des droits à l'échelle de la planète. Les défenseurs d'une perspective particulariste ne sont pas opposés à la justice globale et ils s'entendront avec les cosmopolites de la seconde catégorie. Mais pas avec les premiers. Non pas parce qu'il sont des partisans de l'État et du système étatique actuel, mais parce qu'ils pensent que l'appartenance nationale a, dans la majorité des cas, une valeur pour les personnes, que les nations, lorsqu'elles sont libérales, constituent un cadre de vie qui assure le développement de leurs capacités morales et que les solidarités qu'elles suscitent est un facteur important de la démocratie. Selon eux, une nation doit, pour toutes ces raisons, posséder les institutions qui lui permettent de s'autodéterminer et d'orchestrer son évolution. Dans une perspective particulariste, l'État est un modèle d'organisation sociale et politique qui n'est ni parfait ni immuable, et on peut légitimement chercher à en découvrir un meilleur. Mais pour l'instant c'est encore le seul dont nous disposions qui puisse permettre aux nations de jouer le rôle qu'elle peuvent jouer pour leurs membres.

Les travaux portant sur le cosmopolitisme institutionnel première manière sont importants mais encore trop peu diversifiés pour nous permettre de juger s'il y a de ce côté quelque perspective d'avenir. Quant à la seconde manière, elle nous semble pour l'instant plus prometteuse pour autant que, s'appuyant sur des structures institutionnelles déjà en place, elle permette de concevoir avec précision les mécanismes qui permettront effectivement de satisfaire, dans une certaine mesure, l'idéal d'égalité cosmopolitique. Comme nous avons pu le constater dans cette introduction, la question de la nature des institutions requises et celle de la nature et de l'objet des obligations cosmopolitiques risquent d'alimenter le débat pendant quelques années encore.

\section{Bibliographie}

Appiah, Kwame Anthony. « Cosmopolitan Patriots » in P. Cheah et Bruce Robbins (dir.), Cosmopolitics, University of Minnesota Press, 91-114, 1998.

- The Ethics of Identity, Princeton University Press, 2005.

Barry, Brian. «Statism and Nationalism : a Cosmopolitan Culture» in I. Shapiro et L. Brilmayer (dir.), Global Justice, New York University Press, 1999.

Beitz, Charles. «Philosophy of International Relations ». In Routledge Encyclopaedia of Philosophy, Routledge, 826-33, 1998.

Beitz, Charles. Political Theory and International Relations (seconde édition), Princeton University Press, 1997. 
Cohen, Mitchell. «Rooted Cosmopolitanism». In Michael Walzer (dir.), Toward a Global Civil Society, Berghahn Books, 223-33, 1995.

Couture, Jocelyne. "Cosmopolitan Democracy and Liberal Nationalism ", The Monist, vol. 82, $\mathrm{n}^{\circ}$ 3, 491-515, 1999. Repris in Nenad Miscevic (dir.), Nationalism and Ethnic Conflict, Chicago, Open Court, 261-282, 2000.

—. "Nationalisme et démocratie mondiale. Entre le mythe de la communauté et le mirage du village global ». In Michel Seymour (dir.), États-nations, multinations et organisations internationales, Montréal, Liber, 205-226, 2002. Repris sous le titre "Nationalism and Global Democracy: Between the Myth of the Community and the Mirage of the Global Village », dans Michel Seymour (dir), The Fate of the Nation-State, Montréal: McGill / Kingston: Queen's University Press, 69-89, 2004.

—. "Domination et Distribution », Geneviève Nootens (dir.), Bulletin d'histoire politique, vol. 12, $\mathrm{n}^{\circ}$ 3, «Diversité, mondialisation et justice. La philosophie politique devant les grands enjeux contemporains », 2004.

Couture, Jocelyne et Kai Nielsen. "Cosmopolitanism and the Compatriot Priority Principle », H. Brighouse et G. Brook (dir.), The Political Philosophy of Cosmopolitism, Cambridge, Cambridge University Press, 180-195, 2005.

Habermas, Jürgen. The Postnational Constellation, The MIT Press, 2001.

Held, David. Democracy and the Global Order, Cambridge University Press, 1995. Kymlicka, Will. Liberalism, Community and Culture, Clarendon Press, 1989.

Miller, David. «Cosmopolitanism : A Critique », CRISPP, vol. 5, n 3, 80-85, 2002.

Nielsen, Kai. "Cosmopolitanism, Universalism and Particularism in an Age of Nationalism and Multiculturalism », Philosophical Exchange, $\mathrm{n}^{\circ} 29,1998$.

—. "Cosmopolitan Nationalism ", The Monist, vol. 82, n 3, 446-469, 1999. Repris in Nenad Miscevic (dir.), Nationalism and Ethnic Conflict, Chicago, Open Court, 2000.

Nussbaum, Martha. For the Love of Country, Beacon Press, 2002.

«Beyond the Social Contract: Capabilities and Global Justice». In G. Brook et H. Brighouse (dir.), The Political Philosophy of Cosmopolitism, Cambridge University Press, 2005.

Pogge, Thomas. "An Egalitarian Law of Peoples ", Philosophy and Public Affairs, vol. 23, 195-224, 1994.

- World Poverty and Human Rights, Polity, 2002a.

—. "Cosmopolitanism: A Defense », CRISPP, vol. 5, n 3, 86-91, 2002 b.

Rawls, John. The Law of Peoples, Harvard University Press, 1999.

Scheffler, Samuel. Boundaries of Allegiances, Oxford Universal Press, 2001.

Tan, Kok-Chor. Toleration, Diversity, and Global Justice, The Pennsylvania State University Press, 2000. 